\title{
A Multiscale Butterfly Algorithm for Multidimensional Fourier Integral Operators
}

\author{
Yingzhou Li $i^{\sharp}$, Haizhao Yang ${ }^{\dagger *}$ and Lexing Ying ${ }^{\dagger}$ \\ $\dagger$ Department of Mathematics, Stanford University \\ \# ICME, Stanford University
}

Nov 2014

\begin{abstract}
This paper presents an efficient multiscale butterfly algorithm for computing Fourier integral operators (FIOs) of the form $(\mathcal{L} f)(x)=\int_{\mathbb{R}^{d}} a(x, \xi) e^{2 \pi \imath \Phi(x, \xi)} \widehat{f}(\xi) d \xi$, where $\Phi(x, \xi)$ is a phase function, $a(x, \xi)$ is an amplitude function, and $f(x)$ is a given input. The frequency domain is hierarchically decomposed into a union of Cartesian coronas. The integral kernel $a(x, \xi) e^{2 \pi \imath \Phi(x, \xi)}$ in each corona satisfies a special low-rank property that enables the application of a butterfly algorithm on the Cartesian phase-space grid. This leads to an algorithm with quasi-linear operation complexity and linear memory complexity. Different from previous butterfly methods for the FIOs, this new approach is simple and reduces the computational cost by avoiding extra coordinate transformations. Numerical examples in two and three dimensions are provided to demonstrate the practical advantages of the new algorithm.
\end{abstract}

Keywords. Fourier integral operators, the butterfly algorithm, hierarchical decomposition, separated representation.

AMS subject classifications: 44A55, 65R10 and 65T50.

\section{Introduction}

This paper is concerned with the rapid application of Fourier integral operators (FIOs), which are defined as

$$
(\mathcal{L} f)(x)=\int_{\mathbb{R}^{d}} a(x, \xi) e^{2 \pi \imath \Phi(x, \xi)} \widehat{f}(\xi) d \xi
$$

where

- $a(x, \xi)$ is an amplitude function that is smooth both in $x$ and $\xi$,

- $\Phi(x, \xi)$ is a phase function that is smooth in $(x, \xi)$ for $\xi \neq 0$ and obeys the homogeneity condition of degree 1 in $\xi$, namely, $\Phi(x, \lambda \xi)=\lambda \Phi(x, \xi)$ for each $\lambda>0$;

- $\widehat{f}$ is the Fourier transform of the input $f$ defined by

$$
\widehat{f}(\xi)=\int_{\mathbb{R}^{d}} e^{-2 \pi \imath x \cdot \xi} f(x) d x .
$$

\footnotetext{
${ }^{*}$ Corresponding author. Email address: haizhao@math.stanford.edu.
} 
The computation of Fourier integral operators appears quite often in the numerical solution of wave equations and related applications in computational geophysics. In a typical setting, it is often assumed that the problem is periodic (i.e., $a(x, \xi), \Phi(x, \xi)$, and $f(x)$ are all periodic in $x$ ) or the function $f(x)$ decays sufficiently fast so that one can embed the problem in a sufficiently large periodic cell. A simple discretization in two dimensions considers functions $f$ given on a Cartesian grid

$$
X=\left\{x=\left(\frac{n_{1}}{N}, \frac{n_{2}}{N}\right), 0 \leq n_{1}, n_{2}<N \text { with } n_{1}, n_{2} \in \mathbb{Z}\right\}
$$

in a unit square and defines the discrete Fourier integral operator by

$$
(L f)(x)=\sum_{\xi \in \Omega} a(x, \xi) e^{2 \pi \imath \Phi(x, \xi)} \widehat{f}(\xi), \quad x \in X,
$$

where

$$
\Omega=\left\{\xi=\left(n_{1}, n_{2}\right),-\frac{N}{2} \leq n_{1}, n_{2}<\frac{N}{2} \text { with } n_{1}, n_{2} \in \mathbb{Z}\right\}
$$

and $\widehat{f}$ is the discrete Fourier transform of $f$

$$
\widehat{f}(\xi)=\frac{1}{N^{2}} \sum_{x \in X} e^{-2 \pi \imath x \cdot \xi} f(x) .
$$

In most examples, since $a(x, \xi)$ is a smooth symbol of order zero and type $(1,0)$ [3, 6, 14, 17, 24, $a(x, \xi)$ is numerically low-rank in the joint $X$ and $\Omega$ domain and its numerical treatment is relatively easy. Therefore, we will simplify the problem by assuming $a(x, \xi)=1$ in the following analysis and the algorithm description. [7] is referred to for discussion on how to deal with a non-constant amplitude function. Under this assumption, the discrete FIO discussed in this paper takes the following form:

$$
(L f)(x)=\sum_{\xi \in \Omega} e^{2 \pi \imath \Phi(x, \xi)} \widehat{f}(\xi), \quad x \in X .
$$

A direct computation of (4) takes $\mathcal{O}\left(N^{4}\right)$ operations, which is quadratic in the number of DOFs $N^{2}$. Hence, a practical need is to design efficient and accurate algorithms to evaluate (4). This research topic is of great interest for computing wave equations especially in geophysics [15, 18, 23, 25].

\section{$1.1 \quad$ Previous work}

An earlier method for the rapid computation of general FIOs is the algorithm for two dimensional problems proposed in [6]. This method starts by partitioning the frequency domain $\Omega$ into $\mathcal{O}(\sqrt{N})$ wedges of equal angle. The integral (4) restricted to each wedge is then factorized into two components, both of which can be handled efficiently. The first one has a low-rank structure that leads to an $\mathcal{O}\left(N^{2} \log N\right)$ fast computation, while the second one is a non-uniform Fourier transform which can be evaluated in $\mathcal{O}\left(N^{2} \log N\right)$ steps with the algorithms developed in [1, 16, 22]. Summing the computational cost over all $\mathcal{O}(\sqrt{N})$ wedges gives an $\mathcal{O}\left(N^{2.5} \log N\right)$ computational cost.

Shortly after, an algorithm with quasilinear complexity for general FIOs was proposed in [7] using the framework of the butterfly algorithms in [20, 21]. This approach introduces a polar coordinate transformation in the frequency domain to remove the singularity of $\Phi(x, \xi)$ at $\xi=0$, proves the existence of low-rank separated approximations between certain pairs of spatial and frequency domains, and implements the low-rank approximations with oscillatory Chebyshev interpolations. The resulting algorithm evaluates (4) with $\mathcal{O}\left(N^{2} \log N\right)$ operations and $\mathcal{O}\left(N^{2}\right)$ memory, both essentially linear in terms of the number of unknowns. 
Another related research direction seeks for sparse representations of the FIOs using modern basis functions from harmonic analysis. A sparse representation allows fast matrix-vector products in the transformed domain. Local Fourier transforms [2, 4, 11, wavelet-packet transforms [19], the curvelet transform [5, 8, 9, 10], and the wave atom frame [12, 13] have been investigated for the purpose of operator sparsification. In spite of favorable asymptotic behaviors, the actual representations of the FIOs typically have a large pre-factor constant in terms of both the computational time and the memory requirement. This makes them less competitive compared to the approaches in [6, 7].

\subsection{Motivation}

The main motivation of the current work is to improve the performance of the butterfly algorithm in [7]. As we pointed out earlier, this algorithm starts by applying a polar coordinate transformation in the frequency domain to remove the singularity of the phase function at $\xi=0$. For this reason, we refer to this algorithm as the polar Butterfly algorithm. More precisely, the polar butterfly algorithm introduces a polar-Cartesian coordinate transformation $T:\left(p_{1}, p_{2}\right) \rightarrow\left(\xi_{1}, \xi_{2}\right)$ such that

$$
\xi=\left(\xi_{1}, \xi_{2}\right)=\frac{\sqrt{2}}{2} N p_{1} e^{2 \pi \imath p_{2}}, \quad e^{2 \pi \imath p_{2}}=\left(\cos 2 \pi p_{2}, \sin 2 \pi p_{2}\right) .
$$

Let $P=T^{-1}(\Omega)$. By definition, each point $p=\left(p_{1}, p_{2}\right) \in P$ belongs to $[0,1]^{2}$. The new phase function $\Psi(x, p)$ in the $p$ variable is now given by

$$
\Psi(x, p):=\frac{1}{N} \Phi(x, \xi)=\frac{\sqrt{2}}{2} \Phi\left(x, e^{2 \pi \imath p_{2}}\right) p_{1},
$$

where the last identity comes from the homogeneity of $\Phi(x, \xi)$ in $\xi$. Thus, computing (44) is equivalent to evaluate

$$
(L f)(x)=\sum_{\xi \in \Omega} e^{2 \pi \imath \Phi(x, \xi)} \widehat{f}(\xi)=\sum_{p \in P} e^{2 \pi \imath N \Psi(x, p)} \widehat{f}(T(p)) .
$$

The new phase function $\Psi(x, p)$ is smooth in the whole domain $(x, p) \subset[0,1]^{2} \times[0,1]^{2}$, since $\Phi(x, \xi)$ is smooth in $(x, \xi)$ for $\xi \neq 0$. This smoothness guarantees a low-rank separated approximation of

$e^{2 \pi \imath N \Psi(x, p)}$ when $x$ and $p$ are properly restricted to certain subdomains in $X \times P$ under certain geometric configuration. This low-rank property allows for the application of the butterfly algorithm in [26] and results in a fast algorithm with an $\mathcal{O}\left(N^{2} \log N\right)$ computational complexity and an $\mathcal{O}\left(N^{2}\right)$ memory complexity.

However, the application of this polar-Cartesian transformation comes with several drawbacks, which result in a large pre-factor of the computational complexity. First, due to the polar grid in the frequency domain, the points in $P$ for the butterfly algorithm are irregularly distributed and a separate Chebyshev interpolation matrix is required for the evaluation at each point. In order to avoid the memory bottleneck from storing these interpolation matrices, the polar butterfly algorithm generates these interpolation matrices on-the-fly during the evaluation. This turns out to be expensive in the operation count. Second, since the amplitude and phase functions are often written in the Cartesian coordinates, the polar butterfly algorithm applies the polar-Cartesian transformation for each kernel evaluation. Finally, in order to maintain a reasonable accuracy, the polar butterfly algorithm divides the frequency domain into multiple parts and applies the same butterfly algorithm to each part separately. This also increases the actual running time by a non-trivial constant factor. 


\subsection{Our contribution}

Those drawbacks of the polar Butterfly algorithm motivate us to propose a multiscale butterfly algorithm using a Cartesian grid both in the spatial and frequency domain. To deal with the singularity of the kernel $\Phi(x, \xi)$ at $\xi=0$, we hierarchically decompose the frequency domain into a union of non-overlapping Cartesian coronas with a common center $\xi=0$ (see Figure 11). More precisely, define

$$
\Omega_{j}=\left\{\left(n_{1}, n_{2}\right): \frac{N}{2^{j+1}}<\max \left(\left|n_{1}\right|,\left|n_{2}\right|\right) \leq \frac{N}{2^{j}}\right\} \cap \Omega
$$

for $j=1, \ldots, \log N-s$, where $s$ is just a small constant integer. The domain $\Omega_{d}=\Omega \backslash \cup_{j} \Omega_{j}$ is the remaining square grid at the center of constant size. Following this decomposition of the frequency domain, one can write (4) accordingly as

$$
(L f)(x)=\sum_{j}\left(\sum_{\xi \in \Omega_{j}} e^{2 \pi \imath \Phi(x, \xi)} \widehat{f}(\xi)\right)+\sum_{\xi \in \Omega_{d}} e^{2 \pi \imath \Phi(x, \xi)} \widehat{f}(\xi) .
$$

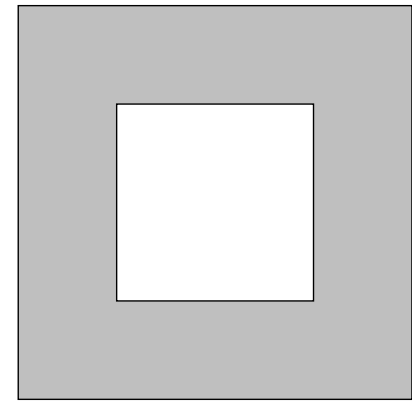

$\Omega_{1}$

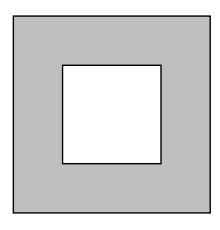

$\Omega_{2}$

$$
\Omega_{\log N-s} \quad \Omega_{d}
$$

Figure 1: This figure shows the frequency domain decomposition of $\Omega$. Each sub-domain $\Omega_{j}$, $j=1, \ldots, \log N-s$, is a corona and $\Omega_{d}$ is a small square domain near the origin.

The kernel function of (8) is smooth in each sub-domain $\Omega_{j}$ and a Cartesian butterfly algorithm is applied to evaluate the contribution from $\Omega_{j}$. For the center square $\Omega_{d}$, since it contains only a constant number of points, a direct summation is used. Because of the mutiscale nature of the frequency domain decompositions, we refer to this algorithm as the multiscale butterfly algorithm. As we shall see, the computational and memory complexity of the multiscale butterfly algorithm are still $\mathcal{O}\left(N^{2} \log N\right)$ and $\mathcal{O}\left(N^{2}\right)$, respectively. On the other hand, the pre-factors are much smaller, since the multiscale butterfly is based on the Cartesian grids and requires no polarCartesian transformation.

\subsection{Organization}

The rest of this paper is organized as follows. Section 2 presents the overall structure of a butterfly algorithm. Section 3 proves a low-rank property that is essential to the multiscale butterfly algorithm. Section 4 combines the results of the previous two sections and describes the multiscale butterfly algorithm in detail. In Section 5, numerical results of several examples are provided to demonstrate the efficiency of the multiscale butterfly algorithm. Finally, we conclude this paper with some discussion in Section 6 . 


\section{The Butterfly Algorithm}

This section provides brief description of the overall structure of the butterfly algorithm. In this section, $X$ and $\Omega$ refer to two general sets of $M$ points in $\mathbb{R}^{2}$, respectively. We assume the points in these two sets are distributed quasi-uniformly but they are not necessarily the sets defined in (2) and (3).

Given an input $\{g(\xi), \xi \in \Omega\}$, the goal is to compute the potentials $\{u(x), x \in X\}$ defined by

$$
u(x)=\sum_{\xi \in \Omega} K(x, \xi) g(\xi), \quad x \in X,
$$

where $K(x, \xi)$ is a kernel function. Let $D_{X} \supset X$ and $D_{\Omega} \supset \Omega$ be two square domains containing $X$ and $\Omega$ respectively. The main data structure of the butterfly algorithm is a pair of quadtrees $T_{X}$ and $T_{\Omega}$. Having $D_{X}$ as its root box, the tree $T_{X}$ is built by recursive dyadic partitioning of $D_{X}$ until each leaf box contains only a few points. The tree $T_{\Omega}$ is constructed by recursively partitioning in the same way. With the convention that a root node is at level 0 , a leaf node is at level $L=O(\log M)$ under the quasi-uniformity condition about the point distributions, where $M$ is the number of points in $X$ and $\Omega$. Throughout, we shall use $A$ and $B$ to denote the square boxes of $T_{X}$ and $T_{\Omega}$ with $\ell_{A}$ and $\ell_{B}$ denoting their levels, respectively.

At the heart of the butterfly algorithm is a special low-rank property. Consider any pair of boxes $A \in T_{X}$ and $B \in T_{\Omega}$ obeying the condition $\ell_{A}+\ell_{B}=L$. The butterfly algorithm assumes that the submatrix $\{K(x, \xi)\}_{x \in A, \xi \in B}$ to be approximately of a constant rank. More precisely, for

any $\epsilon$, there exists a constant $r_{\epsilon}$ independent of $M$ and two sets of functions $\left\{\alpha_{t}^{A B}(x)\right\}_{1 \leq t \leq r_{\epsilon}}$ and $\left\{\beta_{t}^{A B}(\xi)\right\}_{1 \leq t \leq r_{\epsilon}}$ such that the following holds

$$
\left|K(x, \xi)-\sum_{t=1}^{r_{\epsilon}} \alpha_{t}^{A B}(x) \beta_{t}^{A B}(\xi)\right| \leq \epsilon, \quad \forall x \in A, \forall \xi \in B .
$$

The number $r_{\epsilon}$ is called the $\epsilon$-separation rank. The exact form of the functions $\left\{\alpha_{t}^{A B}(x)\right\}_{1 \leq t \leq r_{\epsilon}}$ and $\left\{\beta_{t}^{A B}(\xi)\right\}_{1 \leq t \leq r_{\epsilon}}$ of course depends on the problem to which the butterfly algorithm is applied.

For a given square $B$ in $D_{\Omega}$, define $u^{B}(x)$ to be the restricted potential over the sources $\xi \in B$

$$
u^{B}(x)=\sum_{\xi \in B} K(x, \xi) g(\xi)
$$

The low-rank property gives a compact expansion for $\left\{u^{B}(x)\right\}_{x \in A}$ as summing (9) over $\xi \in B$ with weights $g(\xi)$ gives

$$
\left|u^{B}(x)-\sum_{t=1}^{r_{\epsilon}} \alpha_{t}^{A B}(x)\left(\sum_{\xi \in B} \beta_{t}^{A B}(\xi) g(\xi)\right)\right| \leq\left(\sum_{\xi \in B}|g(\xi)|\right) \epsilon, \quad \forall x \in A .
$$

Therefore, if one can find coefficients $\left\{\delta_{t}^{A B}\right\}_{1 \leq t \leq r_{\epsilon}}$ obeying

$$
\delta_{t}^{A B} \approx \sum_{\xi \in B} \beta_{t}^{A B}(\xi) g(\xi), \quad 1 \leq t \leq r_{\epsilon}
$$

then the restricted potential $\left\{u^{B}(x)\right\}_{x \in A}$ admits a compact expansion

$$
\left|u^{B}(x)-\sum_{t=1}^{r_{\epsilon}} \alpha_{t}^{A B}(x) \delta_{t}^{A B}\right| \leq\left(\sum_{\xi \in B}|g(\xi)|\right) \epsilon, \quad \forall x \in A .
$$


A key point of the butterfly algorithm is that for each pair $(A, B)$, the number of terms in the expansion is independent of $M$.

Computing $\left\{\delta_{t}^{A B}\right\}_{1 \leq t \leq r_{\epsilon}}$ by means of (10) for all pairs $A, B$ is not efficient when $B$ is a large box because for each $B$ there are many paired boxes $A$. The butterfly algorithm, however, comes with an efficient way for computing $\left\{\delta_{t}^{A B}\right\}_{1 \leq t \leq r_{\epsilon}}$ recursively. The general structure of the algorithm consists of a top down traversal of $T_{X}$ and a bottom up traversal of $T_{\Omega}$, carried out simultaneously.

1. Construct the trees $T_{X}$ and $T_{\Omega}$ with root nodes $D_{X}$ and $D_{\Omega}$.

2. Let $A$ be the root of $T_{X}$. For each leaf box $B$ of $T_{\Omega}$, construct the expansion coefficients $\left\{\delta_{t}^{A B}\right\}_{1 \leq t \leq r_{\epsilon}}$ for the potential $\left\{u^{B}(x)\right\}_{x \in A}$ by simply setting

$$
\delta_{t}^{A B}=\sum_{\xi \in B} \beta_{t}^{A B}(\xi) g(\xi), \quad 1 \leq t \leq r_{\epsilon} .
$$

3. For $\ell=1,2, \ldots, L$, visit level $\ell$ in $T_{X}$ and level $L-\ell$ in $T_{\Omega}$. For each pair $(A, B)$ with $\ell_{A}=\ell$ and $\ell_{B}=L-\ell$, construct the expansion coefficients $\left\{\delta_{t}^{A B}\right\}_{1 \leq t \leq r_{\epsilon}}$ for the potential $\left\{u^{B}(x)\right\}_{x \in A}$ using the low-rank representation constructed at the previous level $(\ell=0$ is the initialization step). Let $P$ be $A$ 's parent and $C$ be a child of $B$. Throughout, we shall use the notation $C \succ B$ when $C$ is a child of $B$. At level $\ell-1$, the expansion coefficients $\left\{\delta_{s}^{P C}\right\}_{1 \leq s \leq r_{\epsilon}}$ of $\left\{u^{C}(x)\right\}_{x \in P}$ are readily available and we have

$$
\left|u^{C}(x)-\sum_{s=1}^{r_{\epsilon}} \alpha_{s}^{P C}(x) \delta_{s}^{P C}\right| \leq\left(\sum_{\xi \in C}|g(\xi)|\right) \epsilon, \quad \forall x \in P .
$$

Since $u^{B}(x)=\sum_{C \succ B} u^{C}(x)$, the previous inequality implies that

$$
\left|u^{B}(x)-\sum_{C \succ B} \sum_{s=1}^{r_{\epsilon}} \alpha_{s}^{P C}(x) \delta_{s}^{P C}\right| \leq\left(\sum_{\xi \in B}|g(\xi)|\right) \epsilon, \quad \forall x \in P .
$$

Since $A \subset P$, the above approximation is of course true for any $x \in A$. However, since $\ell_{A}+$ $\ell_{B}=L$, the sequence of restricted potentials $\left\{u^{B}(x)\right\}_{x \in A}$ also has a low-rank approximation of size $r_{\epsilon}$, namely,

$$
\left|u^{B}(x)-\sum_{t=1}^{r_{\epsilon}} \alpha_{t}^{A B}(x) \delta_{t}^{A B}\right| \leq\left(\sum_{\xi \in B}|g(\xi)|\right) \epsilon, \quad \forall x \in A .
$$

Combining the last two approximations, we obtain that $\left\{\delta_{t}^{A B}\right\}_{1 \leq t \leq r_{\epsilon}}$ should obey

$$
\sum_{t=1}^{r_{\epsilon}} \alpha_{t}^{A B}(x) \delta_{t}^{A B} \approx \sum_{C \succ B} \sum_{s=1}^{r_{\epsilon}} \alpha_{s}^{P C}(x) \delta_{s}^{P C}, \quad \forall x \in A .
$$

This is an over-determined linear system for $\left\{\delta_{t}^{A B}\right\}_{1 \leq t \leq r_{\epsilon}}$ when $\left\{\delta_{s}^{P C}\right\}_{1<s \leq r_{\epsilon}, C \succ B}$ are available. Instead of computing $\left\{\delta_{t}^{A B}\right\}_{1 \leq t \leq r_{\epsilon}}$ with a least-square method, the butterfly algorithm typically uses an efficient linear transformation approximately mapping $\left\{\delta_{s}^{P C}\right\}_{1 \leq s \leq r_{\epsilon}, C \succ B}$ into $\left\{\delta_{t}^{A B}\right\}_{1 \leq t \leq r_{\epsilon}}$. The actual implementation of this step is very much application-dependent. 


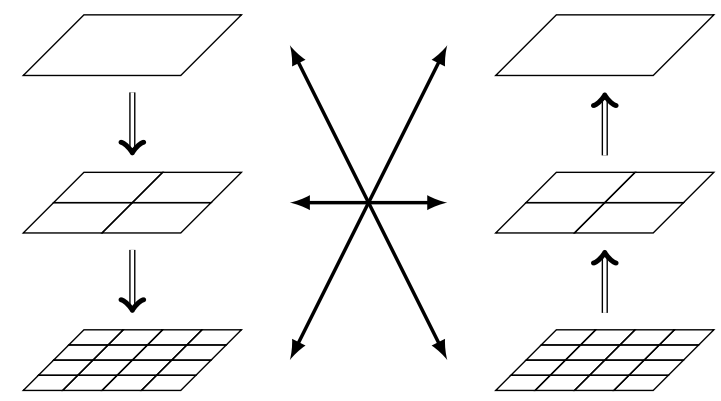

Figure 2: Hierarchical domain trees of the 2D butterfly algorithm. Left: $T_{X}$ for the spatial domain $D_{X}$. Right: $T_{\Omega}$ for the frequency domain $D_{\Omega}$. The interactions between subdomains $A \subset D_{X}$ and $B \subset D_{\Omega}$ are represented by left right arrow lines.

4. Finally, $\ell=L$ and set $B$ to be the root node of $T_{\Omega}$. For each leaf box $A \in T_{X}$, use the constructed expansion coefficients $\left\{\delta_{t}^{A B}\right\}_{1 \leq t \leq r_{\epsilon}}$ to evaluate $u(x)$ for each $x \in A$,

$$
u(x)=\sum_{t=1}^{r_{\epsilon}} \alpha_{t}^{A B}(x) \delta_{t}^{A B} .
$$

A schematic illustration of this algorithm is provided in Figure 2. We would like to emphasize that the strict balance between the levels of the target boxes $A$ and source boxes $B$ maintained throughout this procedure is the key to obtain the accurate low-rank separated approximations.

\section{Low-rank approximations}

In this section, the set $X$ and $\Omega$ refer to the sets defined in (21) and (3). In order to apply the algorithm in Section 4, one would require the existence of the following low-rank separated representation

$$
e^{2 \pi \imath \Phi(x, \xi)} \approx \sum_{t=1}^{r_{\epsilon}} \alpha_{t}^{A B}(x) \beta_{t}^{A B}(\xi)
$$

for any pair of boxes $A$ and $B$ such that $\ell_{A}+\ell_{B}=L$. However, this is not true for a general FIO kernel $e^{2 \pi \imath \Phi(x, \xi)}$ due to the singularity of $\Phi(x, \xi)$ at the origin $\xi=0$, i.e., when the square $B$ in $\Omega$ is close to the origin of the frequency domain. However, if the frequency domain $B$ is well-separated from the origin $\xi=0$ in a relative sense, one can prove a low-rank separated representation.

In order to make it more precise, for two given squares $A \subset X$ and $B \subset \Omega$, we introduce a new function called the residue phase function

$$
R^{A B}(x, \xi):=\Phi(x, \xi)-\Phi\left(c_{A}, \xi\right)-\Phi\left(x, c_{B}\right)+\Phi\left(c_{A}, c_{B}\right),
$$

where $c_{A}$ and $c_{B}$ are the centers of $A$ and $B$ respectively. Using this new definition, the kernel can be written as

$$
e^{2 \pi \imath \Phi(x, \xi)}=e^{2 \pi \imath \Phi\left(c_{A}, \xi\right)} e^{2 \pi \imath \Phi\left(x, c_{B}\right)} e^{-2 \pi \imath \Phi\left(c_{A}, c_{B}\right)} e^{2 \pi \imath R^{A B}(x, \xi)} .
$$

Theorem 3.1. Suppose $\Phi(x, \xi)$ is a phase function that is real analytic for $x$ and $\xi$ away from $\xi=0$. There exists positive constants $\epsilon_{0}$ and $N_{0}$ such that the following is true. Let $A$ and $B$ be 
two squares in $X$ and $\Omega$, respectively, obeying $w_{A} w_{B} \leq 1$ and $\operatorname{dist}(B, 0) \geq \frac{N}{4}$. For any positive $\epsilon \leq \epsilon_{0}$ and $N \geq N_{0}$, there exists an approximation

$$
\left|e^{2 \pi \imath R^{A B}(x, \xi)}-\sum_{t=1}^{r_{\epsilon}} \tilde{\alpha}_{t}^{A B}(x) \tilde{\beta}_{t}^{A B}(\xi)\right| \leq \epsilon
$$

for $x \in A$ and $\xi \in B$ with $r_{\epsilon} \lesssim \log ^{4}\left(\frac{1}{\epsilon}\right)$. Moreover,

- when $w_{B} \leq \sqrt{N}$, the functions $\left\{\tilde{\beta}_{t}^{A B}(\xi)\right\}_{1 \leq t \leq r_{\epsilon}}$ can all be chosen as monomials in $\left(\xi-c_{B}\right)$ with a degree not exceeding a constant times $\log ^{2}(1 / \epsilon)$,

- and when $w_{A} \leq 1 / \sqrt{N}$, the functions $\left\{\tilde{\alpha}_{t}^{A B}(x)\right\}_{1 \leq t \leq r_{\epsilon}}$ can all be chosen as monomials in $\left(x-c_{A}\right)$ with a degree not exceeding a constant times $\log ^{2}(1 / \epsilon)$.

In Theorem 3.1, $w_{A}$ and $w_{B}$ denote the side lengths of $A$ and $B$, respectively; $\operatorname{dist}(B, 0)$ denotes the distance between the square $B$ and the origin 0 in the frequency domain. The distance is given by $\operatorname{dist}(B, 0)=\min _{\xi \in B}\|\xi-0\|$. Throughout this paper, when we write $\mathcal{O}(\cdot), \lesssim$ and $\gtrsim$, the implicit constant is independent of $N$ and $\epsilon$.

Proof. Since $w_{A} w_{B} \leq 1$, we either have $w_{A} \leq 1 / \sqrt{N}$ or $w_{B} \leq \sqrt{N}$ or both.

Let us first consider the case $w_{B} \leq \sqrt{N}$. Then

$$
\begin{aligned}
R^{A B}(x, \xi) & =\Phi(x, \xi)-\Phi\left(c_{A}, \xi\right)-\Phi\left(x, c_{B}\right)+\Phi\left(c_{A}, c_{B}\right) \\
& =\left[\Phi(x, \xi)-\Phi\left(c_{A}, \xi\right)\right]-\left[\Phi\left(x, c_{B}\right)-\Phi\left(c_{A}, c_{B}\right)\right] \\
& =H(x, \xi)-H\left(x, c_{B}\right)
\end{aligned}
$$

where $H(x, \xi):=\Phi(x, \xi)-\Phi\left(c_{A}, \xi\right)$. The function $R^{A B}(x, \xi)$ inherits the smoothness from $\Phi(x, \xi)$. Applying the multi-variable Taylor expansion of degree $k$ in $\xi$ centered at $c_{B}$ gives

$$
R^{A B}(x, \xi)=\sum_{1 \leq|i|<k} \frac{\partial_{\xi}^{i} H\left(x, c_{B}\right)}{i !}\left(\xi-c_{B}\right)^{i}+\sum_{|i|=k} \frac{\partial_{\xi}^{i} H\left(x, \xi^{*}\right)}{i !}\left(\xi-c_{B}\right)^{i}
$$

where $\xi^{*}$ is a point in the segment between $c_{B}$ and $\xi$. Here $i=\left(i_{1}, i_{2}\right)$ is a multi-index with $i !=i_{1} ! i_{2}$ !, and $|i|=i_{1}+i_{2}$. Let us first choose the degree $k$ so that the second sum in (16) is bounded by $\epsilon /(4 \pi)$. For each $i$ with $|i|=k$, the definition of $H(x, \xi)$ gives

$$
\partial_{\xi}^{i} H\left(x, \xi^{*}\right)=\sum_{|j|=1} \partial_{x}^{j} \partial_{\xi}^{i} \Phi\left(x^{*}, \xi^{*}\right)\left(x-c_{A}\right)^{j}
$$

for some point $x^{*}$ in the segment between $c_{A}$ and $x$. Using the fact that $\Phi(x, \xi)$ is real-analytic over $|\xi|=1$ gives that there exists a radius $R$ such that

$$
\left|\partial_{x}^{j} \partial_{\xi}^{i} \Phi(x, \xi)\right| \leq C i ! j ! \frac{1}{R^{|i+j|}}=C i ! j ! \frac{1}{R^{k+1}},
$$

for $\xi$ with $|\xi|=1$. Here the constant $C$ is independent of $k$. Since $\Phi(x, \xi)$ is homogeneous of degree 1 in $\xi$, a scaling argument shows that

$$
\left|\partial_{x}^{j} \partial_{\xi}^{i} \Phi\left(x^{*}, \xi^{*}\right)\right| \leq C i ! j ! \frac{1}{R^{k+1}\left|\xi^{*}\right|^{k-1}} .
$$


Since $\operatorname{dist}(B, 0) \geq N / 4$ and $w_{A} w_{B} \leq 1$, we have

$$
\left|\frac{\partial_{\xi}^{i} H\left(x, \xi^{*}\right)}{i !}\left(\xi-c_{B}\right)^{i}\right| \leq \frac{2 C i ! j !}{i !} \frac{1}{R^{k+1}\left|\xi^{*}\right|^{k-1}} w_{A} w_{B}^{k} \leq \frac{2 C}{R^{k+1}}\left(\frac{4}{\sqrt{N}}\right)^{k-1} .
$$

Combining this with (16) gives

$$
\left|R^{A B}(x, \xi)-\sum_{1 \leq|i|<k} \frac{\partial_{\xi}^{i} H\left(x, c_{B}\right)}{i !}\left(\xi-c_{B}\right)^{i}\right|=\left|\sum_{|i|=k} \frac{\partial_{\xi}^{i} H\left(x, \xi^{*}\right)}{i !}\left(\xi-c_{B}\right)^{i}\right| \leq \frac{2 C(k+1)}{R^{k+1}}\left(\frac{4}{\sqrt{N}}\right)^{k-1} .
$$

Therefore, for a sufficient large $N_{0}(R)$, if $N>N_{0}(R)$, choosing $k=k_{\epsilon}=O(\log (1 / \epsilon))$ ensures that the difference is bounded by $\epsilon /(4 \pi)$.

The special case $k=1$ results in the following bound for $R^{A B}(x, \xi)$

$$
\left|R^{A B}(x, \xi)\right| \leq \frac{4 C}{R^{2}}
$$

To simplify the notation, we define

$$
R_{\epsilon}^{A B}(x, \xi):=\sum_{1 \leq|i|<k_{\epsilon}} \frac{\partial_{\xi}^{i} H\left(x, c_{B}\right)}{i !}\left(\xi-c_{B}\right)^{i}
$$

i.e., the first sum on the right hand side of (16) with $k=k_{\epsilon}$. The choice of $k_{\epsilon}$ together with (16) implies the bound

$$
\left|R_{\epsilon}^{A B}(x, \xi)\right| \leq \frac{4 C}{R^{2}}+\epsilon
$$

Since $R_{\epsilon}^{A B}(x, \xi)$ is bounded, a direct application of Lemma 3.2 of [7] gives

$$
\left|e^{2 \pi \imath R_{\epsilon}^{A B}(x, \xi)}-\sum_{p=0}^{d_{\epsilon}} \frac{\left(2 \pi \imath R_{\epsilon}^{A B}(x, \xi)\right)^{p}}{p !}\right| \leq \epsilon / 2
$$

where $d_{\epsilon}=\mathcal{O}(\log (1 / \epsilon))$. Since $R_{\epsilon}^{A B}(x, \xi)$ is a polynomial in $\left(\xi-c_{B}\right)$, the sum in (17) is also a polynomial in $\left(\xi-c_{B}\right)$ with degree bounded by $k_{\epsilon} d_{\epsilon}=\mathcal{O}\left(\log ^{2}(1 / \epsilon)\right)$. Since our problem is in 2D, there are at most $\mathcal{O}\left(\log ^{4}(1 / \epsilon)\right)$ possible monomial in $\left(\xi-c_{B}\right)$ with degree bounded by $k_{\epsilon} d_{\epsilon}$. Grouping the terms with the same multi-index in $\xi$ results in an $\mathcal{O}\left(\log ^{4}(1 / \epsilon)\right)$ term $\epsilon$-accurate separated approximation for $e^{2 \pi \imath R_{\epsilon}^{A B}(x, \xi)}$ with the factors $\left\{\tilde{\beta}_{t}^{A B}(\xi)\right\}_{1 \leq t \leq r_{\epsilon}}$ being monomials of $\left(\xi-c_{B}\right)$.

Finally, from the inequality $\left|e^{\imath a}-e^{\imath b}\right| \leq|a-b|$, it is clear that a separated approximation for $e^{2 \pi \imath R_{\epsilon}^{A B}(x, \xi)}$ with accuracy $\epsilon / 2$ is also one for $e^{2 \pi \imath R^{A B}(x, \xi)}$ with accuracy $\epsilon / 2+\epsilon / 2=\epsilon$. This completes the proof for the case $w_{B} \leq \sqrt{N}$.

The proof for the case $w_{A} \leq 1 / \sqrt{N}$ is similar. The only difference is that we now group with

$$
R^{A B}(x, \xi)=\left[\Phi(x, \xi)-\Phi\left(x, c_{B}\right)\right]-\left[\Phi\left(c_{A}, \xi\right)-\Phi\left(c_{A}, c_{B}\right)\right]
$$

and apply the multivariable Taylor expansion in $x$ centered at $c_{A}$ instead. This results an $\mathcal{O}\left(\log ^{4}(1 / \epsilon)\right)$ term $\epsilon$-accurate separated approximation for $e^{2 \pi \imath R^{A B}(x, \xi)}$ with the factors $\left\{\tilde{\alpha}_{t}^{A B}(x)\right\}_{1 \leq t \leq r_{\epsilon}}$ being monomials of $\left(x-c_{A}\right)$. 
Though the above proof is constructive, it is cumbersome to construct the separated approximation this way. On the other hand, the proof shows that when $w_{B} \leq \sqrt{N}$, the $\xi$-dependent factors in the low-rank approximation of $e^{2 \pi \imath R^{A B}(x, \xi)}$ are all monomials in $\left(\xi-c_{B}\right)$. Similarly, when $w_{A} \leq 1 / \sqrt{N}$, the $x$-dependent factors are monomials in $\left(x-c_{A}\right)$. This suggests to use Chebyshev interpolation in $x$ when $w_{A} \leq 1 / \sqrt{N}$ and in $\xi$ when $w_{B} \leq \sqrt{N}$. For this purpose, we associate with each box a Chebyshev grid as follows.

For a fixed integer $q$, the Chebyshev grid of order $q$ on $[-1 / 2,1 / 2]$ is defined by

$$
\left\{z_{i}=\frac{1}{2} \cos \left(\frac{i \pi}{q-1}\right)\right\}_{0 \leq i \leq q-1} .
$$

A tensor-product grid adapted to a square with center $c$ and side length $w$ is then defined via shifting and scaling as

$$
\left\{c+w\left(z_{i}, z_{j}\right)\right\}_{i, j=0,1, \ldots, q-1}
$$

In what follows, $M_{t}^{B}$ is the $2 \mathrm{D}$ Lagrange interpolation polynomial on the Chebyshev grid adapted to the square $B$ (i.e., using $c=c_{B}$ and $w=w_{B}$ ).

Theorem 3.2. Let $A$ and $B$ be as in Theorem 3.1. Then for any $\epsilon \leq \epsilon_{0}$ and $N \geq N_{0}$ where $\epsilon_{0}$ and $N_{0}$ are the constants in Theorem [3.1, there exists $q_{\epsilon} \lesssim \log ^{2}(1 / \epsilon)$ such that

- when $w_{B} \leq \sqrt{N}$, the Lagrange interpolation of $e^{2 \pi \imath R^{A B}(x, \xi)}$ in $\xi$ on a $q_{\epsilon} \times q_{\epsilon}$ Chebyshev grid $\left\{g_{t}^{B}\right\}_{1 \leq t \leq r_{\epsilon}}$ adapted to $B$ obeys

$$
\left|e^{2 \pi \imath R^{A B}(x, \xi)}-\sum_{t=1}^{r_{\epsilon}} e^{2 \pi \imath R^{A B}\left(x, g_{t}^{B}\right)} M_{t}^{B}(\xi)\right| \leq \epsilon, \quad \forall x \in A, \forall \xi \in B,
$$

- when $w_{A} \leq 1 / \sqrt{N}$, the Lagrange interpolation of $e^{2 \pi \imath R^{A B}(x, \xi)}$ in $x$ on a $q_{\epsilon} \times q_{\epsilon}$ Chebyshev grid $\left\{g_{t}^{A}\right\}_{1 \leq t \leq r_{\epsilon}}$ adapted to A obeys

$$
\left|e^{2 \pi \imath R^{A B}(x, \xi)}-\sum_{t=1}^{r_{\epsilon}} M_{t}^{A}(x) e^{2 \pi \imath R^{A B}\left(g_{t}^{A}, \xi\right)}\right| \leq \epsilon, \quad \forall x \in A, \forall \xi \in B .
$$

Both (18) and (19) provide a low-rank approximation with $r_{\epsilon}=q_{\epsilon}^{2} \lesssim \log ^{4}(1 / \epsilon)$ terms.

The proof for this follows exactly the one of Theorem 3.3 of [7].

Finally, we are ready to construct the low-rank approximation for the kernel $e^{2 \pi \imath \Phi(x, \xi)}$, i.e.,

$$
e^{2 \pi \imath \Phi(x, \xi)} \approx \sum_{t=1}^{r_{\epsilon}} \alpha_{t}^{A B}(x) \beta_{t}^{A B}(\xi) .
$$

When $w_{B} \leq \sqrt{N}$, one multiply (18) with $e^{2 \pi \imath \Phi\left(c_{A}, \xi\right)} e^{2 \pi \imath \Phi\left(x, c_{B}\right)} e^{-2 \pi \imath \Phi\left(c_{A}, c_{B}\right)}$, which gives that $\forall x \in$ $A, \forall \xi \in B$

$$
\left|e^{2 \pi \imath \Phi(x, \xi)}-\sum_{t=1}^{r_{\epsilon}} e^{2 \pi \imath \Phi\left(x, g_{t}^{B}\right)}\left(e^{-2 \pi \imath \Phi\left(c_{A}, g_{t}^{B}\right)} M_{t}^{B}(\xi) e^{2 \pi \imath \Phi\left(c_{A}, \xi\right)}\right)\right| \leq \epsilon .
$$

In terms of the notations in (20), the expansion functions are given by

$$
\alpha_{t}^{A B}(x)=e^{2 \pi \imath \Phi\left(x, g_{t}^{B}\right)}, \quad \beta_{t}^{A B}(\xi)=e^{-2 \pi \imath \Phi\left(c_{A}, g_{t}^{B}\right)} M_{t}^{B}(\xi) e^{2 \pi \imath \Phi\left(c_{A}, \xi\right)}, \quad 1 \leq t \leq r_{\epsilon} .
$$


This is a special interpolant of the function $e^{2 \pi \imath \Phi(x, \xi)}$ in the $\xi$ variable, which pre-factors the oscillation, performs the interpolation, and then remodulates the outcome. When $w_{A} \leq 1 / \sqrt{N}$, multiply (19) with $e^{2 \pi \imath \Phi\left(c_{A}, \xi\right)} e^{2 \pi \imath \Phi\left(x, c_{B}\right)} e^{-2 \pi \imath \Phi\left(c_{A}, c_{B}\right)}$ and obtain that $\forall x \in A, \forall \xi \in B$

$$
\left|e^{2 \pi \imath \Phi(x, \xi)}-\sum_{t=1}^{r_{\epsilon}}\left(e^{2 \pi \imath \Phi\left(x, c_{B}\right)} M_{t}^{A}(x) e^{-2 \pi \imath \Phi\left(g_{t}^{A}, c_{B}\right)}\right) e^{2 \pi \imath \Phi\left(g_{t}^{A}, \xi\right)}\right| \leq \epsilon .
$$

The expansion functions are now

$$
\alpha_{t}^{A B}(x)=e^{2 \pi \imath \Phi\left(x, c_{B}\right)} M_{t}^{A}(x) e^{-2 \pi \imath \Phi\left(g_{t}^{A}, c_{B}\right)}, \quad \beta_{t}^{A B}(\xi)=e^{2 \pi \imath \Phi\left(g_{t}^{A}, \xi\right)}, \quad 1 \leq t \leq r_{\epsilon} .
$$

Due to the presence of the demodulation and remodulation steps in the definitions (21) and (22), we refer to them as oscillatory Chebyshev interpolations.

\section{Multiscale Butterfly Algorithm}

In this section, we combine the low-rank approximations described in Section 3 with the butterfly algorithm in Section 2. Due to the restriction on the distance between $B$ and the origin, we decompose (44) into a multiscale summation

$$
(L f)(x)=\sum_{\xi \in \Omega_{d}} e^{2 \pi \imath \Phi(x, \xi)} \widehat{f}(\xi)+\sum_{j} \sum_{\xi \in \Omega_{j}} e^{2 \pi \imath \Phi(x, \xi)} \widehat{f}(\xi),
$$

where

$$
\Omega_{j}=\left\{\left(n_{1}, n_{2}\right): \frac{N}{2^{j+1}}<\max \left(\left|n_{1}\right|,\left|n_{2}\right|\right) \leq \frac{N}{2^{j}}\right\} \cap \Omega
$$

for $j=1, \ldots, \log N-s, s$ is a constant, and $\Omega_{d}=\Omega \backslash \cup_{j} \Omega_{j}$.

The term of $\Omega_{d}$ can be evaluated directly since $\left|\Omega_{d}\right|=\mathcal{O}(1)$. Let us now fix an $\Omega_{j}$. Since any square $B$ in $\Omega_{j}$ always stays away from the origin, the results in Section 3 applies to the term for $\Omega_{j}$ in (23). Therefore, the butterfly algorithm as described in Section 2 can be adapted to evaluate

$$
\sum_{\xi \in \Omega_{j}} e^{2 \pi \imath \Phi(x, \xi)} \widehat{f}(\xi)
$$

for the Cartesian domains $X$ and $\Omega_{j}$. In contrast to the polar Butterfly algorithm that works in the polar coordinates for $\Omega$, we refer to this one as the Cartesian butterfly algorithm.

\subsection{Cartesian butterfly algorithm}

To make it more explicit, let us first consider the interaction between $\left(X, \Omega_{1}\right)$, with the low-rank approximation implemented using the oscillatory Chebyshev interpolation discussed in Section 3 .

1. Preliminaries. Construct two quadtrees $T_{X}$ and $T_{\Omega_{1}}$ for $X$ and $\Omega_{1}$ by uniform hierarchical partitioning. Let $b$ be a constant greater than or equal to 4 and define $N_{1}=N$.

2. Initialization. For each square $A \in T_{X}$ of width $1 / b$ and each square $B \in T_{\Omega_{1}}$ of width $b$, the low-rank approximation functions are

$$
\alpha_{t}^{A B}(x)=e^{2 \pi \imath \Phi\left(x, g_{t}^{B}\right)}, \quad \beta_{t}^{A B}(\xi)=e^{-2 \pi \imath \Phi\left(c_{A}, g_{t}^{B}\right)} M_{t}^{B}(\xi) e^{2 \pi \imath \Phi\left(c_{A}, \xi\right)}, \quad 1 \leq t \leq r_{\epsilon} .
$$

Hence, we can define the expansion weights $\left\{\delta_{t}^{A B}\right\}_{1 \leq t \leq r_{\epsilon}}$ with

$$
\delta_{t}^{A B}:=\sum_{\xi \in B} \beta_{t}^{A B}(\xi) \widehat{f}(\xi)=e^{-2 \pi \imath \Phi\left(c_{A}, g_{t}^{B}\right)} \sum_{\xi \in B}\left(M_{t}^{B}(\xi) e^{2 \pi \imath \Phi\left(c_{A}, \xi\right)} \widehat{f}(\xi)\right) .
$$


3. Recursion. Go up in tree $T_{\Omega_{1}}$ and down in tree $T_{X}$ at the same time until we reach the level such that $w_{B}=\sqrt{N_{1}}$. At each level, visit all the pairs $(A, B)$. We apply the Chebyshev interpolation in variable $\xi$ and still define the approximation functions given in (24). Let $\left\{\delta_{s}^{P C}\right\}_{1 \leq s \leq r_{\epsilon}}$ denote the expansion coefficients available in previous steps, where $P$ is $A$ 's parent, $C$ is a child of $B$, and $s$ indicates the Chebyshev grid points in previous domain pairs. We define the new expansion coefficients $\left\{\delta_{t}^{A B}\right\}_{1 \leq t \leq r_{\epsilon}}$ as

$$
\delta_{t}^{A B}:=e^{-2 \pi \imath \Phi\left(c_{A}, g_{t}^{B}\right)} \sum_{C \succ B} \sum_{s=1}^{r_{\epsilon}} M_{t}^{B}\left(g_{s}^{C}\right) e^{2 \pi \imath \Phi\left(c_{A}, g_{s}^{C}\right)} \delta_{s}^{P C},
$$

where we recall that the notation $C \succ B$ means that $C$ is a child of $B$.

4. Switch. For the levels visited, the Chebyshev interpolation is applied in variable $\xi$, while the interpolation is applied in variable $x$ for levels $l>\log \left(N_{1}\right) / 2$. Hence, we are switching the interpolation method at this step. Now we are still working on level $l=\log \left(N_{1}\right) / 2$ and the same domain pairs $(A, B)$ in the last step. Let $\delta_{s}^{A B}$ denote the expansion weights obtained by Chebyshev interpolation in variable $\xi$ in the last step. Correspondingly, $\left\{g_{s}^{B}\right\}_{s}$ are the grid points in $B$ in the last step. We take advantage of the interpolation in variable $x$ in $A$ and generate grid points $\left\{g_{t}^{A}\right\}_{1 \leq t \leq r_{\epsilon}}$ in $A$. Then we can define new expansion weights

$$
\delta_{t}^{A B}:=\sum_{s=1}^{r_{\epsilon}} e^{2 \pi \imath \Phi\left(g_{t}^{A}, g_{s}^{B}\right)} \delta_{s}^{A B}
$$

5. Recursion. Go up in tree $T_{\Omega_{1}}$ and down in tree $T_{X}$ at the same time until we reach the level such that $w_{B}=N_{1} / b$. We construct the approximation functions by Chebyshev interpolation in variable $x$ as follows:

$$
\alpha_{t}^{A B}(x)=e^{2 \pi \imath \Phi\left(x, c_{B}\right)} M_{t}^{A}(x) e^{-2 \pi \imath \Phi\left(g_{t}^{A}, c_{B}\right)}, \quad \beta_{t}^{A B}(\xi)=e^{2 \pi \imath \Phi\left(g_{t}^{A}, \xi\right)} .
$$

We define the new expansion coefficients $\left\{\delta_{t}^{A B}\right\}_{1 \leq t \leq r_{\epsilon}}$ as

$$
\delta_{t}^{A B}:=\sum_{C \succ B} e^{2 \pi \imath \Phi\left(g_{t}^{A}, c_{C}\right)} \sum_{s=1}^{r_{\epsilon}}\left(M_{s}^{P}\left(g_{t}^{A}\right) e^{-2 \pi \imath \Phi\left(g_{s}^{P}, c_{C}\right)} \delta_{s}^{P C}\right),
$$

where again $P$ is $A$ 's parent and $C$ is a child box of $B$.

6. Termination. Finally, we reach the level that $w_{B}=N_{1} / b$. For each $B$ on this level and for each square $A \in T_{X}$ of width $b / N_{1}$, we apply the approximation functions given by (27) and obtain

$$
u^{B}(x):=e^{2 \pi \imath \Phi\left(x, c_{B}\right)} \sum_{t=1}^{r_{\epsilon}}\left(M_{t}^{A}(x) e^{-2 \pi \imath \Phi\left(g_{t}^{A}, c_{B}\right)} \delta_{t}^{A B}\right)
$$

for each $x \in A$. Finally, summing over all $B$ on this level, we have

$$
u^{\Omega_{1}}(x):=\sum_{B} u^{B}(x)
$$

for each $x \in A$. 
We would like to emphasize that the center part of the tree $T_{\Omega_{j}}$ is always empty since $\Omega_{j}$ is a corona. Accordingly, the algorithm skips this empty part.

For a general $\Omega_{j}$, the interaction between $\left(X, \Omega_{j}\right)$ follows a similar algorithm, except that we replace $\Omega_{1}$ with $\Omega_{j}, u^{\Omega_{1}}(x)$ with $u^{\Omega_{j}}(x), N_{1}$ with $N_{j}=N / 2^{j-1}$, and stop at the level that $w_{B}=N_{j} / b$.

Finally, (23) is evaluated via

$$
(L f)(x)=u^{\Omega_{d}}(x)+\sum_{j} u^{\Omega_{j}}(x) .
$$

\subsection{Complexity analysis}

The cost of evaluating the term of $\Omega_{d}$ takes at most $\mathcal{O}\left(N^{2}\right)$ steps since $\left|\Omega_{d}\right|=\mathcal{O}(1)$. Let us now consider the cost of the terms associated with $\left\{\Omega_{j}\right\}$.

For the interaction between $X$ and $\Omega_{1}$, the computation consists of two parts: the recursive evaluation of $\left\{\delta_{t}^{A B}\right\}$ and the final evaluation of $u^{\Omega_{1}}(x)$. The recursive part takes $\mathcal{O}\left(q^{3} N^{2} \log N\right)$ since there are at most $\mathcal{O}\left(N^{2} \log N\right)$ pairs of squares $(A, B)$ and the evaluation of $\left\{\delta_{t}^{A B}\right\}$ for each pair takes $\mathcal{O}\left(q^{3}\right)$ steps via dimension-wise Chebyshev interpolation. The final evaluation of $u^{\Omega_{1}}(x)$ clearly takes $\mathcal{O}\left(q^{2} N^{2}\right)$ steps as we spend $\mathcal{O}\left(q^{2}\right)$ on each point $x \in X$.

For the interaction between $X$ and $\Omega_{j}$, the analysis is similar. The recursive part takes now $\mathcal{O}\left(q^{3} N_{j}^{2} \log N_{j}\right)$ steps (with $N_{j}=N / 2^{j-1}$ ) as there are at most $\mathcal{O}\left(N_{j}^{2} \log N_{j}\right)$ pairs of squares involved. The final evaluation still takes $\mathcal{O}\left(q^{2} N^{2}\right)$ steps.

Summing these contributions together results in the total computational complexity

$$
\mathcal{O}\left(q^{3} N^{2} \log N\right)+\mathcal{O}\left(q^{2} N^{2} \log N\right)=\mathcal{O}\left(q^{3} N^{2} \log N\right)=\mathcal{O}\left(r_{\epsilon}^{3 / 2} N^{2} \log N\right) .
$$

The multiscale butterfly algorithm is also highly efficient in terms of memory as the Cartesian butterfly algorithm is applied sequentially to evaluate (29) for each $\Omega_{j}$. The overall memory complexity is $O\left(\frac{N^{2}}{b^{2}}\right)$, only $\frac{1}{b^{2}}$ of that the original Cartesian butterfly algorithm.

\section{Numerical results}

This section presents several numerical examples to demonstrate the effectiveness of the multiscale butterfly algorithm introduced above. In truth, FIOs usually have non-constant amplitude functions. Nevertheless, the main computational difficulty is the oscillatory phase term. We refer to [7] for detailed fast algorithms to deal with non-constant amplitude functions. Our MATLAB implementation can be found on the authors' personal homepages. The numerical results were obtained on a desktop with a $3.5 \mathrm{GHz} \mathrm{CPU}$ and $32 \mathrm{~GB}$ of memory. Let $\left\{u^{d}(x), x \in X\right\},\left\{u^{m}(x), x \in X\right\}$ and $\left\{u^{p}(x), x \in X\right\}$ be the results of a discrete FIO computed by a direct matrix-vector multiplication, the multiscale butterfly algorithm and the polar butterfly algorithm [7], respectively. To report on the accuracy, we randomly select a set $S$ of 256 points from $X$ and evaluate the relative errors of the multiscale butterfly algorithm and the polar butterfly algorithm by

$$
\epsilon^{m}=\sqrt{\frac{\sum_{x \in S}\left|u^{d}(x)-u^{m}(x)\right|^{2}}{\sum_{x \in S}\left|u^{d}(x)\right|^{2}}} \text { and } \epsilon^{p}=\sqrt{\frac{\sum_{x \in S}\left|u^{d}(x)-u^{p}(x)\right|^{2}}{\sum_{x \in S}\left|u^{d}(x)\right|^{2}}} .
$$

According to the description of the multiscale butterfly algorithm in Section 4 , we recursively divide $\Omega$ into $\Omega_{j}, j=1,2, \ldots, \log N-s$, where $s$ is 5 in the following examples. This means that the center square $\Omega_{d}$ is of size $2^{5} \times 2^{5}$ and the interaction from $\Omega_{d}$ is evaluated via a direct matrix-vector 
multiplication. Suppose $q_{\epsilon}$ is the number of Chebyshev points in each dimension. There is no sense to use butterfly algorithms to construct $\left\{\delta_{t}^{A B}\right\}$ when the number of points in $B$ is fewer than $q_{\epsilon}^{2}$. Hence, the recursion step in butterfly algorithms starts from the squares $B$ that are a couple of levels away from the bottom of $T_{\Omega}$ such that each square contains at least $q_{\epsilon}^{2}$ points. Similarly, the recursion stops at the squares in $T_{X}$ that are the same number of levels away from the bottom. In the following examples, we start from level $\log N-3$ and stop at level 3 (corresponding to $b=2^{3}$ defined in Section (4) which matches with $q_{\epsilon}(4$ to 11$)$.

In order to make a fair comparison, we compare the MATLAB versions of the polar butterfly algorithm and the multiscale butterfly algorithm. Hence, the running time of the polar butterfly algorithm here is slower than the one in [7], which was implemented in $\mathrm{C}++$.

Example 1. The first example is a generalized Radon transform whose kernel is given by

$$
\begin{aligned}
\Phi(x, \xi) & =x \cdot \xi+\sqrt{c_{1}^{2}(x) \xi_{1}^{2}+c_{2}^{2}(x) \xi_{2}^{2}}, \\
c_{1}(x) & =\left(2+\sin \left(2 \pi x_{1}\right) \sin \left(2 \pi x_{2}\right)\right) / 3 \\
c_{2}(x) & =\left(2+\cos \left(2 \pi x_{1}\right) \cos \left(2 \pi x_{2}\right)\right) / 3 .
\end{aligned}
$$

We assume the amplitude of this example is a constant 1. Now the FIO models an integration over ellipses where $c_{1}(x)$ and $c_{2}(x)$ are the axis lengths of the ellipse centered at the point $x \in X$. Table 1

\begin{tabular}{|c|c|c|c|c|c|c|}
\hline \multicolumn{3}{|c|}{ Multiscale Butterfly } & \multicolumn{3}{|c|}{ Polar Butterfly } & \\
\hline$N, q_{\epsilon}$ & $\epsilon^{m}$ & $T_{m}(s e c)$ & $N, q_{\epsilon}$ & $\epsilon^{p}$ & $T_{p}(\sec )$ & $T_{p} / T_{m}$ \\
\hline 256,5 & $7.89 \mathrm{e}-02$ & $6.96 \mathrm{e}+01$ & 256,5 & $4.21 \mathrm{e}-02$ & $4.84 \mathrm{e}+02$ & $6.96 \mathrm{e}+00$ \\
\hline 512,5 & $9.01 \mathrm{e}-02$ & $3.62 \mathrm{e}+02$ & 512,5 & $5.54 \mathrm{e}-02$ & $2.34 \mathrm{e}+03$ & $6.46 \mathrm{e}+00$ \\
\hline 1024,5 & $9.13 \mathrm{e}-02$ & $1.81 \mathrm{e}+03$ & 1024,5 & $4.26 \mathrm{e}-02$ & $1.14 \mathrm{e}+04$ & $6.31 \mathrm{e}+00$ \\
\hline 2048,5 & $9.47 \mathrm{e}-02$ & $8.79 \mathrm{e}+03$ & 2048,5 & - & - & - \\
\hline 256,7 & $6.95 \mathrm{e}-03$ & $8.20 \mathrm{e}+01$ & 256,7 & $5.66 \mathrm{e}-03$ & $5.97 \mathrm{e}+02$ & $7.28 \mathrm{e}+00$ \\
\hline 512,7 & $8.43 \mathrm{e}-03$ & $4.16 \mathrm{e}+02$ & 512,7 & $5.89 \mathrm{e}-03$ & $2.82 \mathrm{e}+03$ & $6.79 \mathrm{e}+00$ \\
\hline 1024,7 & $8.45 \mathrm{e}-03$ & $2.03 \mathrm{e}+03$ & 1024,7 & $4.84 \mathrm{e}-03$ & $1.35 \mathrm{e}+04$ & $6.64 \mathrm{e}+00$ \\
\hline 2048,7 & $8.42 \mathrm{e}-03$ & $1.04 \mathrm{e}+04$ & 2048,7 & - & - & - \\
\hline 256,9 & $3.90 \mathrm{e}-04$ & $1.10 \mathrm{e}+02$ & 256,9 & $8.25 \mathrm{e}-04$ & $7.74 \mathrm{e}+02$ & $7.04 \mathrm{e}+00$ \\
\hline 512,9 & $3.42 \mathrm{e}-04$ & $5.39 \mathrm{e}+02$ & 512,9 & $6.78 \mathrm{e}-04$ & $3.57 \mathrm{e}+03$ & $6.61 \mathrm{e}+00$ \\
\hline 1024,9 & $7.61 \mathrm{e}-04$ & $2.74 \mathrm{e}+03$ & 1024,9 & $4.18 \mathrm{e}-04$ & $1.67 \mathrm{e}+04$ & $6.09 \mathrm{e}+00$ \\
\hline 2048,9 & $4.82 \mathrm{e}-04$ & $1.25 \mathrm{e}+04$ & 2048,9 & - & - & - \\
\hline 256,11 & $2.15 \mathrm{e}-05$ & $1.84 \mathrm{e}+02$ & 256,11 & $3.69 \mathrm{e}-05$ & $1.15 \mathrm{e}+03$ & $6.27 \mathrm{e}+00$ \\
\hline 512,11 & $1.89 \mathrm{e}-05$ & $8.60 \mathrm{e}+02$ & 512,11 & $5.53 \mathrm{e}-05$ & $5.10 \mathrm{e}+03$ & $5.93 \mathrm{e}+00$ \\
\hline 1024,11 & $1.96 \mathrm{e}-05$ & $4.27 \mathrm{e}+03$ & 1024,11 & $2.042 \mathrm{e}-05$ & $2.30 \mathrm{e}+04$ & $5.39 \mathrm{e}+00$ \\
\hline 2048,11 & $1.50 \mathrm{e}-05$ & $1.82 \mathrm{e}+04$ & 2048,11 & - & - & - \\
\hline
\end{tabular}
summarize the results of this example given by the polar butterfly algorithm and the multiscale butterfly algorithm.

Table 1: Comparison of the multiscale butterfly algorithm and the polar butterfly algorithm for the phase function in (33). $T_{m}$ is the running time of the multiscale butterfly algorithm; $T_{a}$ is the running time of the polar butterfly algorithm; and $T_{m} / T_{p}$ is the speedup factor.

Example 2. Next, we provide an FIO example with a smooth amplitude function,

$$
u(x)=\sum_{\xi \in \Omega} a(x, \xi) e^{2 \pi \imath \Phi(x, \xi)} \widehat{f}(\xi)
$$


where the amplitude and phase functions are given by

$$
\begin{gathered}
a(x, \xi)=\left(J_{0}(2 \pi \rho(x, \xi))+\imath Y_{0}(2 \pi \rho(x, \xi))\right) e^{-\pi \imath \rho(x, \xi),} \\
\Phi(x, \xi)=x \cdot \xi+\rho(x, \xi), \\
\rho(x, \xi)=\sqrt{c_{1}^{2}(x) \xi_{1}^{2}+c_{2}^{2}(x) \xi_{2}^{2}}, \\
c_{1}(x)=\left(2+\sin \left(2 \pi x_{1}\right) \sin \left(2 \pi x_{2}\right)\right) / 3, \\
c_{2}(x)=\left(2+\cos \left(2 \pi x_{1}\right) \cos \left(2 \pi x_{2}\right)\right) / 3 .
\end{gathered}
$$

Here, $J_{0}$ and $Y_{0}$ are Bessel functions of the first and second kinds. We refer to [6] for more details of the derivation of these formulas. As discussed in [7, we compute the low rank approximation of the amplitude functions $a(x, \xi)$ first:

$$
a(x, \xi) \approx \sum_{t=1}^{s_{\epsilon}} g_{t}(x) h_{t}(\xi) .
$$

In the second step, we apply the multiscale butterfly algorithm to compute

$$
u_{t}(x)=\sum_{\xi \in \Omega} e^{2 \pi \imath \Phi(x, \xi)} \widehat{f}(\xi) h_{t}(\xi)
$$

and sum up all $g_{t}(x) u_{t}(x)$ to evaluate

$$
u(x)=\sum_{t} g_{t}(x) u_{t}(x)
$$

Table 2 summarizes the results of this example given by the direct method and the multiscale butterfly algorithm.

\begin{tabular}{|rcccc|}
\hline$N, q_{\epsilon}$ & $\epsilon^{m}$ & $T_{d}($ sec $)$ & $T_{m}($ sec $)$ & $T_{d} / T_{m}$ \\
\hline 256,7 & $5.10 \mathrm{e}-03$ & $3.78 \mathrm{e}+03$ & $6.07 \mathrm{e}+02$ & $6.23 \mathrm{e}+00$ \\
512,7 & $7.29 \mathrm{e}-03$ & $3.71 \mathrm{e}+04$ & $3.50 \mathrm{e}+03$ & $1.06 \mathrm{e}+01$ \\
1024,7 & $6.16 \mathrm{e}-03$ & $6.42 \mathrm{e}+05$ & $1.70 \mathrm{e}+04$ & $3.77 \mathrm{e}+01$ \\
\hline 256,9 & $4.49 \mathrm{e}-04$ & $2.34 \mathrm{e}+03$ & $7.88 \mathrm{e}+02$ & $2.97 \mathrm{e}+00$ \\
512,9 & $4.04 \mathrm{e}-04$ & $3.66 \mathrm{e}+04$ & $4.64 \mathrm{e}+03$ & $7.90 \mathrm{e}+00$ \\
1024,9 & $3.88 \mathrm{e}-04$ & $6.21 \mathrm{e}+05$ & $2.17 \mathrm{e}+04$ & $2.86 \mathrm{e}+01$ \\
\hline 256,11 & $1.86 \mathrm{e}-05$ & $2.48 \mathrm{e}+03$ & $1.33 \mathrm{e}+03$ & $1.86 \mathrm{e}+00$ \\
512,11 & $1.80 \mathrm{e}-05$ & $3.60 \mathrm{e}+04$ & $6.94 \mathrm{e}+03$ & $5.18 \mathrm{e}+00$ \\
1024,11 & $2.39 \mathrm{e}-05$ & $5.96 \mathrm{e}+05$ & $2.83 \mathrm{e}+04$ & $2.11 \mathrm{e}+01$ \\
\hline
\end{tabular}

Table 2: Numerical results given by the multiscale butterfly algorithm for the FIO in (34). $T_{d}$ is the running time of the direct evaluation; $T_{m}$ is the running time of the multiscale butterfly algorithm; and $T_{d} / T_{m}$ is the speedup factor.

Note that the accuracy of the multiscale butterfly algorithm is well controlled by the number of Chebyshev points $q_{\epsilon}$. This indicates that our algorithm is numerically stable. Another observation is that the relative error improves on average by a factor of 12 every time $q_{\epsilon}$ is increased by a factor of 2. As we can see in those tables, for a fixed kernel and a fixed $q_{\epsilon}$, the accuracy is almost independent of $N$. Hence, in practical applications, one can increase the value of $q_{\epsilon}$ until a desired accuracy is reached in the problem with a small $N$. In the comparison in Table 1, the 
multiscale butterfly algorithm and the polar butterfly algorithm use $q_{\epsilon}=\{5,7,9,11\}$ and achieve comparable accuracy. Meanwhile, as we observed from Table1, the relative error decreasing rate of the multiscale butterfly algorithm is larger than the decreasing rate of the polar butterfly algorithm. This means if a high accuracy is desired, the multiscale butterfly algorithm requires a smaller $q_{\epsilon}$ to achieve it comparing to the polar butterfly algorithm.

The second concern about the algorithm is the asymptotic complexity. From the $T_{m}$ column of Table 1 and 2, we see that $T_{m}$ almost quadrupled when the problem size doubled under the same $q_{\epsilon}$. According to this, we are convinced that the empirical running time of the multiscale butterfly algorithm follows the $\mathcal{O}\left(N^{2} \log N\right)$ asymptotic complexity. Note that the speedup factor over the polar butterfly algorithm is about 6 and the multiscale butterfly algorithm obtains better accuracy. This makes the multiscale butterfly algorithm quite attractive to practitioners who are interested in evaluating an FIO with a large $N$.

Example 3. Extending the multiscale butterfly algorithm to higher dimensions is straightforward. There are two main modifications: higher dimensional multiscale domain decomposition and Chebyshev interpolation. In three dimensions, the frequency domain is decomposed into cubic shells instead of coronas. The kernel interpolation is applied on a three dimensional Chebyshev grids. We apply our three-dimensional multiscale butterfly algorithm to a simple example integrating over spheres with different radii. We assume a constant amplitude function and the kernel function is given by

$$
\Phi(x, \xi)=x \cdot \xi+c(x) \sqrt{\xi_{1}^{2}+\xi_{2}^{2}}, \quad c(x)=\left(3+\sin \left(2 \pi x_{1}\right) \sin \left(2 \pi x_{2}\right) \sin \left(2 \pi x_{3}\right)\right) / 4 .
$$

Table 3 summarizes the results of this example given by the direct method and the multiscale butterfly algorithm.

\begin{tabular}{|rcccc|}
\hline$N, q_{\epsilon}$ & $\epsilon^{m}$ & $T_{d}(\mathrm{sec})$ & $T_{m}(\mathrm{sec})$ & $T_{d} / T_{m}$ \\
\hline 64,5 & $9.41 \mathrm{e}-02$ & $1.82 \mathrm{e}+04$ & $2.50 \mathrm{e}+03$ & $7.31 \mathrm{e}+00$ \\
128,5 & $7.57 \mathrm{e}-02$ & $6.21 \mathrm{e}+05$ & $2.42 \mathrm{e}+04$ & $2.57 \mathrm{e}+01$ \\
256,5 & $8.23 \mathrm{e}-02$ & $3.91 \mathrm{e}+07$ & $2.35 \mathrm{e}+05$ & $1.66 \mathrm{e}+02$ \\
\hline 64,7 & $1.20 \mathrm{e}-02$ & $1.83 \mathrm{e}+04$ & $7.32 \mathrm{e}+03$ & $2.50 \mathrm{e}+00$ \\
128,7 & $1.03 \mathrm{e}-02$ & $6.03 \mathrm{e}+05$ & $4.48 \mathrm{e}+04$ & $1.35 \mathrm{e}+01$ \\
256,7 & $8.13 \mathrm{e}-03$ & $4.39 \mathrm{e}+07$ & $3.81 \mathrm{e}+05$ & $1.15 \mathrm{e}+02$ \\
\hline
\end{tabular}

Table 3: Numerical results given by the multiscale butterfly algorithm for the phase function in (35).

\section{Conclusion}

A simple and efficient multiscale butterfly algorithm for evaluating FIOs is introduced in this paper. This method hierarchically decomposes the frequency domain into multiscale coronas in order to avoid possible singularity of the phase function $\Phi(x, \xi)$ at $\xi=0$. A Cartesian butterfly algorithm is applied to evaluate the FIO over each corona. Many drawbacks of the original butterfly algorithm based on a polar-Cartesian transform in [7] can be avoided. The new multiscale butterfly algorithm has an $\mathcal{O}\left(N^{2} \log N\right)$ operation complexity with a smaller pre-factor, while keeping the same $\mathcal{O}\left(N^{2}\right)$ memory complexity.

Acknowledgments. This work was partially supported by the National Science Foundation under award DMS-1328230 and the U.S. Department of Energys Advanced Scientific Computing Research program under award DE-FC02-13ER26134/DE-SC0009409. 


\section{References}

[1] C. Anderson and M. D. Dahleh. Rapid computation of the discrete Fourier transform. SIAM J. Sci. Comput., 17(4):913-919, July 1996.

[2] A. Averbuch, E. Braverman, R. Coifman, M. Israeli, and A. Sidi. Efficient computation of oscillatory integrals via adaptive multiscale local Fourier bases. Applied and Computational Harmonic Analysis, 9(1):19 - 53, 2000.

[3] G. Bao and W. Symes. Computation of pseudo-differential operators. SIAM Journal on Scientific Computing, 17(2):416-429, 1996.

[4] B. Bradie, R. Coifman, and A. Grossmann. Fast numerical computations of oscillatory integrals related to acoustic scattering, I. Applied and Computational Harmonic Analysis, 1(1):94 - 99, 1993.

[5] E. Candès, L. Demanet, D. Donoho, and L. Ying. Fast discrete curvelet transforms. Multiscale Model. Simul., 5(3):861-899, 2006.

[6] E. Candès, L. Demanet, and L. Ying. Fast computation of Fourier integral operators. SIAM Journal on Scientific Computing, 29(6):2464-2493, 2007.

[7] E. Candès, L. Demanet, and L. Ying. A fast butterfly algorithm for the computation of Fourier integral operators. Multiscale Modeling and Simulation, 7(4):1727-1750, 2009.

[8] E. Candès and D. L. Donoho. New tight frames of curvelets and optimal representations of objects with piecewise $C^{2}$ singularities. Comm. Pure Appl. Math., 57(2):219-266, 2004.

[9] E. Candès and D. L. Donoho. Continuous curvelet transform. I. Resolution of the wavefront set. Appl. Comput. Harmon. Anal., 19(2):162-197, 2005.

[10] E. Candès and D. L. Donoho. Continuous curvelet transform. II. Discretization and frames. Appl. Comput. Harmon. Anal., 19(2):198-222, 2005.

[11] E. Cordero, F. Nicola, and L. Rodino. Sparsity of Gabor representation of Schrödinger propagators. Applied and Computational Harmonic Analysis, 26(3):357 - 370, 2009.

[12] L. Demanet and L. Ying. Wave atoms and sparsity of oscillatory patterns. Appl. Comput. Harmon. Anal., 23(3):368-387, 2007.

[13] L. Demanet and L. Ying. Scattering in flatland: Efficient representations via wave atoms. Found. Comput. Math., 10(5):569-613, Oct. 2010.

[14] L. Demanet and L. Ying. Discrete symbol calculus. SIAM Review, 53(1):71-104, 2011.

[15] L. Demanet and L. Ying. Fast wave computation via Fourier integral operators. Math. Comput., 81(279), 2012.

[16] A. Dutt and V. Rokhlin. Fast Fourier transforms for nonequispaced data. SIAM Journal on Scientific Computing, 14(6):1368-1393, 1993.

[17] L. Hörmander. Fourier integral operators. I. Acta Mathematica, 127(1):79-183, 1971. 
[18] J. Hu, S. Fomel, L. Demanet, and L. Ying. A fast butterfly algorithm for generalized Radon transforms. Geophysics, 78(4):U41-U51, June 2013.

[19] D. Huybrechs and S. Vandewalle. A two-dimensional wavelet-packet transform for matrix compression of integral equations with highly oscillatory kernel. Journal of Computational and Applied Mathematics, 197(1):218 - 232, 2006.

[20] E. Michielssen and A. Boag. A multilevel matrix decomposition algorithm for analyzing scattering from large structures. Antennas and Propagation, IEEE Transactions on, 44(8):1086-1093, Aug 1996.

[21] M. P. O'Neil. A New Class of Analysis-based Fast Transforms. PhD thesis, New Haven, CT, USA, 2007. AAI3293360.

[22] D. Potts, G. Steidl, and M. Tasche. Fast Fourier transforms for nonequispaced data: A tutorial, 2001.

[23] D. O. Trad, T. J. Ulrych, and M. D. Sacchi. Accurate interpolation with high-resolution time-variant radon transforms. Geophysics, 67(2):644-656, 2002.

[24] H. Yang and L. Ying. A fast algorithm for multilinear operators. Applied and Computational Harmonic Analysis, 33(1):148 - 158, 2012.

[25] B. Yazici, L. Wang, and K. Duman. Synthetic aperture inversion with sparsity constraints. In Electromagnetics in Advanced Applications (ICEAA), 2011 International Conference on, pages 1404-1407, Sept 2011.

[26] L. Ying. Sparse Fourier transform via butterfly algorithm. SIAM J. Sci. Comput., 31(3):16781694, Feb. 2009. 